ISSN: 0213-2087 eISSN: 2444-7080

DOI: https://doi.org/10.14201/shhc202139303324

\title{
EL DIFÍCIL EQUILIBRIO ENTRE LA LEGALIDAD INTERNACIONAL Y LA POLÍTICA EXTERIOR: LA PARTICIPACIÓN DE LAS FUERZAS ARMADAS ESPAÑOLAS EN LA INTERVENCIÓN MILITAR DE LOS ESTADOS UNIDOS EN IRAK EN 2003
}

\author{
The difficult balance between international legality \\ and foreign policy. The participation of the Spanish \\ Armed Forces in the 2003 us military intervention \\ in Iraq
}

José Antonio LORENZO CUESTA

Universidad Nacional de Educación a Distancia (UNED)

https://orcid.org/0000-0003-1100-0498

Recibido: 08/07/2019 Revisado: 09/05/2021 Aceptado: 12/05/2021

RESUMEN: Este artículo pretende demostrar que la participación de las Fuerzas Armadas españolas, llevada a cabo en el año 2003 en el territorio iraquí, se desarrolló en un escenario geoestratégico en el que la Administración norteamericana quiso afianzar su presencia como actor principal en un mundo envuelto en complejas relaciones internacionales y tras un siglo que tanto alumbró la desaparición del comunismo de la Unión Soviética, como asistió a la consolidación del fundamentalismo islámico. Mediante la utilización de los métodos sintético y deductivo, este trabajo llevará a la comprensión del alcance real de la presencia de las tropas españolas en Irak, así como de su verdadero significado político y estratégico. Tras un recorrido por los diferentes epígrafes que conforman este artículo, podrá concluirse que los ciudadanos iraquíes fueron los únicos beneficiados de esta acción, puesto que las relaciones exteriores bilaterales entre España y los Estados Unidos se vieron especialmente afectadas. 


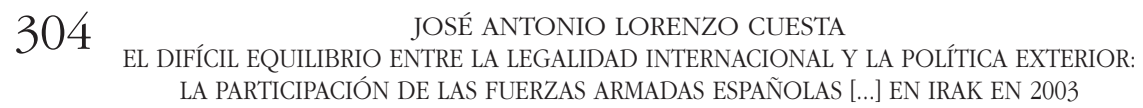

Palabras clave: Política exterior; geoestrategia; legalidad internacional; ayuda humanitaria; guerra; insurgencia.

ABSTRACT: This article aims to demonstrate that the participation of the Spanish Armed Forces, carried out in 2003 in Iraqi territory, took place in a geostrategic scenario in which the us Administration wanted to consolidate its presence as the main actor in a world surrounded by complex international relations and after a century that both illuminated the disappearance of communism in the Soviet Union, as well as the consolidation of Islamic fundamentalism. By using synthetic and deductive methods, this work will lead to an understanding of the real scope of the presence of Spanish troops in Iraq, as well as its true political and strategic significance. After a tour of the different sections that make up this article, it can be concluded that Iraqi citizens were the only beneficiaries of this action, since bilateral foreign relations between Spain and the United States were particularly affected.

Key words: Foreign policy; geostrategy; international legality; humanitarian aid; war; insurgency.

\section{INTRODUCCIÓN}

La crisis y la guerra de Irak han trascendido mediáticamente en pocos países tanto como en España. Durante el primer semestre de 2003, los medios de comunicación centraron su debate en torno a la guerra de Irak desde el punto de vista político, diplomático y del derecho internacional. El discurso se basaba, en todo caso, en el apoyo prestado por el Gobierno de José María Aznar a los planes de invasión estadounidenses. El apoyo incondicional del Gobierno español a la intervención liderada en Irak por los Estados Unidos provocó consecuencias de proporciones políticas y sociales nunca vistas en la política exterior española desde la Transición política, lo que justifica la necesidad de relacionar la participación española en Irak con la nueva estrategia de política exterior norteamericana adoptada tras los acontecimientos del 11S. Asimismo, se destacará el protagonismo adquirido por la Organización de Naciones Unidas (ONU) en este escenario internacional.

Los escasos estudios realizados en España sobre este episodio de su historia se han limitado a profundizar en su mera visión militar. En el plano internacional, muy pocos estudios analizan el juego desarrollado por España sobre el tablero de las relaciones internacionales durante esta etapa, así como la entidad del apoyo prestado a los Estados Unidos en el conflicto con Irak, y ninguno de ellos se ocupa de relacionar estas cuestiones de política internacional con la posición adoptada por España respecto a las resoluciones de la onu. No puede obviarse que el 1 de enero de 2003, España se convirtió en miembro no permanente del Consejo de Seguridad, asiento que ocuparía durante los dos años siguientes. De los 183 Estados parte de la Organización de Naciones Unidas, 180 avalaron la presencia de España en el Consejo de Seguridad, lo que demostraba la confianza depositada en el Estado español y en su quehacer en la esfera internacional. El asunto que monopolizó 


\section{JOSÉ ANTONIO LORENZO CUESTA \\ EL DIFÍCIL EQUILIBRIO ENTRE LA LEGALIDAD INTERNACIONAL Y LA POLÍTICA EXTERIOR: \\ LA PARTICIPACIÓN DE LAS FUERZAS ARMADAS ESPAÑOLAS [...] EN IRAK EN 2003}

la discusión en las primeras reuniones del Consejo de Seguridad del 2003 fue la posibilidad de plantear una guerra inminente contra Irak. Hasta ese momento, España no se había pronunciado explícitamente sobre la aceptación o el rechazo de una intervención militar. La política exterior española primó al vínculo atlántico sobre el europeo y el color político del Gobierno español fue determinante en el grado de participación del Ejército en el conflicto que, a pesar de las controversias suscitadas, ejecutó un programa de ayuda al pueblo iraquí muy beneficioso para la población civil del país árabe.

2. La nueva política geoestratégica de Estados Unidos tras el 11 de SEPTiembre DE 2001 (11S)

Después del 11S, la sociedad americana apostaba por el apoyo a la política defensiva de su Gobierno porque aunque el mundo no había cambiado, sí pudieron apreciarse transformaciones en la percepción de los norteamericanos sobre el mismo y su seguridad ${ }^{1}$. Los atentados del $11 \mathrm{~S}$ no fueron percibidos por los ciudadanos estadounidenses como un ataque a su Administración, sino como un ataque a su forma de concebir el mundo y a la poderosa influencia de su país en ciertos espacios geográficos ${ }^{2}$.

La política exterior de Estados Unidos se había basado históricamente en un complejo equilibrio que la propia Constitución americana contempla, consistente en un entramado de relaciones, checks and balances, capaz de originar unas relaciones internacionales plurales y, en cierto modo, tendentes a la «esquizofrenia» 3 . El presidente, el Congreso, el Departamento de Defensa, el Departamento de Estado, los think tanks privados y públicos, los medios de comunicación y la propia sociedad civil deben de ser oídos en la valoración de las acciones adoptadas en materia de política exterior. El secretario de Estado de Defensa, Donald Rumsfeld, se convenció de que tras los atentados de septiembre de 2001, los 68 planes previstos para la defensa de Estados Unidos ${ }^{4}$ y sus intereses en el mundo debían de ser revisados, comenzando por el denominado Operation Plan 1003, el plan que contemplaba la invasión de Irak.

1. NYE, Joseph S.: La paradoja del poder norteamericano. Madrid: Taurus, 2003, p. 8.

2. Gutiérrez Espada, Cesáreo, González Martín, Andrés: El conflicto de Irak ir. Madrid: Ministerio de Defensa, 2006, p. 8.

3. Waltz, Kenneth N.: Realism and International Politics. Londres: Routledge, 2008, p. 115.

4. Cuatro meses después de los atentados del 11S, Donald Rumsfeld había declarado que la clave contra el terrorismo no era la defensa ante posibles atentados, ya que era imposible defender todos los posibles objetivos e intereses norteamericanos en el mundo. Había que atacar al terrorismo antes de ser atacados. De este modo, nació el concepto fundamental de la nueva Doctrina de Seguridad Nacional de Estados Unidos para el siglo xxI, en septiembre de 2002, el ataque preventivo, la guerra preventiva. WoODWARD, Robert: Plan de ataque. Barcelona: Planeta, 2004, p. 8. 
El 10 de octubre de 2002, el Congreso norteamericano aprobó una resolución 5 que autorizaba al presidente a utilizar las Fuerzas Armadas en Irak si lo consideraba oportuno y necesario. Esta resolución, de acuerdo con el War Power Resolution Act de 1973, autorizaba al presidente a invadir Irak en caso de incumplimiento de las condiciones que la Organización de Naciones Unidas había impuesto a Irak con el fin de destruir sus arsenales de armas de destrucción masiva. No obstante, la resolución aconsejaba que el uso de la fuerza en Irak contase con la aprobación expresa del Consejo de Seguridad de Naciones Unidas, aunque no lo consideraba un requisito de obligado cumplimiento ${ }^{6}$. En este escenario surgió el concepto preemptive, la necesidad de ataques de anticipación. Estados Unidos no solo pretendía justicia, sino que podía permitirse aplicarla por sí mismo. La doctrina de la guerra preventiva, contenida en la Estrategia de Seguridad Nacional de Estados Unidos de América de 2002 y revisada en 2006, afirmaba que el terrorismo y los Estados que intentaran adquirir o producir armas de destrucción masiva constituían los peligros más graves del momento. Para combatir estas amenazas, los Estados Unidos debían identificar y destruir los posibles riesgos, antes de que estos pudieran materializarse en ataques como los ocurridos el 11 de septiembre de 2001. La potencia de las armas de destrucción masiva impedía que Estados Unidos adoptase una política defensiva, por lo que se imponía el concepto de ataque preventivo ${ }^{7}$. Así pues, el objetivo de las acciones preventivas no solo eran los grupos terroristas sino también aquellos Estados que fueran considerados patrocinadores del terrorismo. En este último caso, se justificaba la acción preventiva que podía incluir la acción militar de ocupación del Estado y la renovación de su régimen político. El concepto de acción militar preventiva fue asimilado por otros gobiernos como el del Reino Unido.

Después del 11S, podría aventurarse que Estados Unidos pretendía eliminar a un Estado como el iraquí por representar una amenaza cierta hacia los norteamericanos, debido a su posesión de armas de destrucción masiva. Dada esta primera premisa, podía justificarse el poner fin a esta amenaza latente, bien mediante la eliminación física de Sadam Husein o mediante la invasión de Irak, con la justificación de un ataque preventivo hacia un Estado con un potencial destructivo significativo. Sin embargo, algunos gobiernos y parte de la opinión pública mundial coincidían en que lo que realmente se escondía tras el deseo de atacar Irak era la

5. Fue aprobada con una mayoría más amplia que la que autorizó la liberación de Kuwait en 1991. 296 congresistas votaron a favor, entre ellos 81 demócratas, 133 votaron en contra.

6. En caso de ataque inminente de Irak a Estados Unidos, la resolución consideraba la aprobación del Consejo no imprescindible. La Administración Bush definió la amenaza de Irak como creciente y acumulativa, pero no inminente. MuÑOz, Heraldo: Una Guerra en solitario. Madrid: El tercer hombre editorial, 2006, pp. 85-90.

7. Estrategia de Seguridad Nacional de Estados Unidos de América de 2002, párrafo séptimo. https://georgewbush-whitehouse-archives.gov. El 10 de octubre de 2002 el Senado de Estados Unidos había aprobado por 77 votos a favor y 23 en contra una resolución que otorgaba poderes de guerra al presidente contra Irak. 


\section{JOSÉ ANTONIO LORENZO CUESTA \\ EL DIFÍCIL EQUILIBRIO ENTRE LA LEGALIDAD INTERNACIONAL Y LA POLÍTICA EXTERIOR: \\ LA PARTICIPACIÓN DE LAS FUERZAS ARMADAS ESPAÑOLAS [...] EN IRAK EN 2003}

reafirmación del poder y del dominio de Estados Unidos en Oriente Próximo. Este ataque formaría parte de la estrategia global de proyectar el poder norteamericano a nivel planetario ${ }^{8}$.

Esa mentalidad era la imperante en la Estrategia de Seguridad Nacional de Estados Unidos 9 enviada al Congreso el 20 de septiembre de 2002. Desde el punto de vista geoestratégico, Irak fue presentado como un Estado impredecible, al que no podía permitírsele poseer armas de destrucción masiva porque representaba un peligro para Oriente Próximo y los intereses de Estados Unidos en este territorio. En esta situación, la solución más viable para la Administración Bush consistía en efectuar un ataque preventivo que acabase con el régimen iraquí antes de que dispusiera de capacidad nuclear ${ }^{10}$, además, se apuntaba la inutilidad de las inspecciones de las Naciones Unidas, cuestión en la que el resto de los miembros del Consejo de Seguridad no llegaron al acuerdo. En 1997, se había elaborado el denominado Proyecto para el medio siglo norteamericano, Dick Cheney, Donald Rumsfeld, Paul Wolfowitz y Jeb Bush, entre otros, firmaban este documento que había contado con la colaboración de intelectuales como Francis Fukuyama, Lewis Libby y Norman Podhoretz. El programa basaba su plan de actuación en tres pilares básicos que ya se habían ensayado durante la Administración Reagan: un ejército fuerte preparado para afrontar cualquier desafío presente o futuro, una política exterior encaminada a imponer los principios y valores estadounidenses en el mundo y un liderazgo nacional consciente de la responsabilidad mundial de Estados Unidos ${ }^{11}$. El 12 de septiembre de 2001, el Consejo de Seguridad Nacional

8. Iglesias CAvicchioli, Manuel: «La doctrina neoconservadora y el excepcionalismo americano. Una vía al unilateralismo y a la negación del Derecho Internacional», Revista electrónica de estudios internacionales, 28, 2014, p. 5.

9. Esta nueva concepción estratégica tuvo su antecedente ideológico en la Guía para la Planificación de la Defensa del Pentágono, la llamada doctrina Wolfowitz de mediados de los años noventa del siglo xx. Este documento giraba en torno a la necesidad de evitar la aparición de una potencia capaz de competir con Estados Unidos, utilizando para ello todos los medios posibles a su alcance, incluida la fuerza militar. La estrategia militar estadounidense, ya antes del 11S, contemplaba la disminución de sus fuerzas militares en el este asiático y en Europa, y un incremento de su presencia militar en el Golfo Pérsico y Asia central que se vio acelerado tras los atentados del 11S. Colom, Guillem: De la compensación a la revolución: la configuración de la política de defensa estadounidense contemporánea (1977-2014). Madrid: Instituto Universitario General Gutiérrez Mellado, 2016, pp. 115-117.

10. George J. Tenet, director de la Agencia Central de Inteligencia, había declarado en 2002 que Irak no dispondría de capacidad nuclear hasta la segunda mitad de la década, en el mejor de los casos, y solo material para montar una única bomba atómica. En 1984, tras el bombardeo israelí de las instalaciones nucleares iraquíes de Osirak, Irak había llevado el desarrollo de su programa nuclear dentro del mayor secretismo. En 2002, el régimen de Sadam Husein no había sido capaz de producir el material nuclear necesario para montar un artefacto atómico y tampoco un sistema viable para transportarlo hasta el enemigo. BARDAJí, Rafael: Irak: reflexiones sobre una guerra. Madrid: Real Instituto Elcano, 2003, p. 285.

11. Bacevich, Andrew J.: American Empire: The Realities and Consequences of U.S. Diplomacy. Boston: Harvard University Press, 2002, pp. 243-244. 
de Estados Unidos se reunió para decidir sobre el ataque a Irak o a Afganistán, optándose por este último.

Los objetivos del Proyecto para el medio siglo norteamericano fueron incorporados a la Estrategia Nacional de Seguridad de los Estados Unidos de América que el presidente George W. Bush dio a conocer en septiembre de 2002. El ataque a Irak sería el primer ensayo de esta nueva estrategia. Joseph S. Nye ${ }^{12}$, politólogo estadounidense, reconocía que la política exterior estadounidense no había variado en lo esencial desde la época del presidente Wilson ${ }^{13}$. Para Nye, las corrientes neowilsonianas y neojacksonianas defendían el militarismo apoyado en alianzas a la carta y valiéndose de la legitimidad que otorgan las instituciones internacionales para sacar adelante sus proyectos de política exterior. Para este autor, resultaba más práctico legitimar las actuaciones en el exterior con el consenso previo de los principales actores políticos de la escena internacional. Para ciertos sectores de la opinión pública mundial, Washington habría utilizado el proceso de inspección de las Naciones Unidas ${ }^{14}$ para destruir el régimen de Sadam Husein, con el objetivo de provocar un cambio en Irak que desalojase del poder al Partido Baaz y a su líder.

Sin dudar de la importancia del petróleo ${ }^{15}$ como factor estratégico en los planes estadounidenses en Oriente Próximo, la cuestión del ataque a Irak formaría parte de la estrategia de poder global. La ocupación de Irak y la instauración en ese país de un régimen tutelado por Estados Unidos dejaría a Irán en una situación de cerco por la presencia de bases militares estadounidenses en Asia central en el norte, en Turquía e Irak en el oeste, Kuwait, Arabia Saudí, Qatar y Omán en el sur, y Pakistán y Afganistán en el este. Lograr que Irak formase parte de ese plan geoestratégico posibilitaría a Washington establecer su presencia en Oriente Próximo de una manera mucho más efectiva y global. A largo plazo, la estrategia de la Administración Bush tendría como objetivo el mantenimiento del mundo unipolar surgido tras la desaparición de la Unión Soviética, en el que Estados Unidos no rivalizaría en igualdad.

\section{LA LEGALIDAD INTERNACIONAL}

A finales de 1999, el Consejo de Seguridad decidió sustituir el United Nations Special Commission (unscom), creado por la Resolución 661 (1990) con motivo de

12. Nye, Joseph S.: "U.S. Power and Strategy after Irak», Foreign Affairs, 4, 2003, pp. 62-63.

13. Texto en Harper's Monthly Magazine en 1916 de Mark Twain: "Lo siguiente que harán los hombres de Estado será inventar burdos embustes para atribuir la culpa a la nación que es atacada (...)». Twain, Mark: "The mysterious stranger. A romance (part viI)», Harper's Monthly Magazine, núm. Noviembre, 1916, p. 886.

14. En 1998, las Naciones Unidas estimaban que, como consecuencia del proceso de inspección, se había destruido entre el 90-95 \% de la capacidad iraquí en armamento biológico y químico. Informes del Secretario General presentados al Consejo de Seguridad en 1998, S1998/889.

15. China, Europa y Japón serían cada vez más dependientes del petróleo de Oriente Próximo, una zona bajo influencia de Estados Unidos. 


\section{JOSÉ ANTONIO LORENZO CUESTA \\ EL DIFÍCIL EQUILIBRIO ENTRE LA LEGALIDAD INTERNACIONAL Y LA POLÍTICA EXTERIOR \\ LA PARTICIPACIÓN DE LAS FUERZAS ARMADAS ESPAÑOLAS [...] EN IRAK EN 2003}

la I Guerra del Golfo, por un nuevo órgano, The United Nations Monitoring, Verification and Inspection Commission (UNMOVIC), presidida por el sueco Hans Blix. La Resolución 1284 (1999) exigía a Irak la cooperación con unMOvIC y el Organismo Internacional de la Energía Atómica (OIEA) para lograr la suspensión paulatina del embargo al que el Consejo de Seguridad había sometido al país como consecuencia de la invasión de Kuwait por el régimen de Sadam Husein. En 1990, la Resolución 678 del Consejo de Seguridad había autorizado a una coalición internacional, dirigida por Estados Unidos, a librar una guerra contra Irak para liberar Kuwait. En 2003, la invasión de Irak se llevó a cabo sin la autorización expresa del Consejo de Seguridad a los Estados Unidos y al Reino Unido. El 20 de marzo de 2003, John Dimitri Negroponte, representante permanente de Estados Unidos en el Consejo de Seguridad, justificaba la invasión de Irak sin la debida autorización de la oNU porque la Resolución 687 (1991) imponía una serie de obligaciones a Irak en materia de desarme para establecer el alto el fuego de la I Guerra del Golfo.

Para Estados Unidos, Irak había incumplido de forma reiterada estas condiciones por lo que el alto el fuego quedaba anulado en virtud de la Resolución $687^{16}$ y restablecida la autorización para el uso de la fuerza. La Administración Bush advertía a la ONu de que la inacción ${ }^{17}$ no constituía una opción válida después de una década de intentos vanos por llegar a un acuerdo con Sadam Husein sobre el control de su propio armamento de destrucción masiva. El Consejo de Seguridad, en su Resolución 1441 (2002), ofrecía una última oportunidad a Irak para reconducir la situación por lo que constituía la nueva referencia a tener en cuenta en la valoración del uso de la fuerza, en lugar de la Resolución $687^{18}$. La Resolución 1441 no proporcionaba cobertura jurídica para llevar a cabo una intervención militar en Irak pues, en su párrafo trece, advertía a Irak de que en caso de seguir infringiendo sus obligaciones se exponía a graves consecuencias, pero no mencionaba de forma expresa el empleo

16. COOPER, Richard H.: The responsibility to protect: the global moral compact for the 21st century. Ginebra: Palgrave Macmillan, 2008, p. 47. La Resolución 687 no preveía nada sobre la autorización del uso de la fuerza en Irak.

17. La unsCom había localizado y eliminado cerca de medio millón de litros de agentes químicos varios y casi un millón de litros de agentes precursores necesarios para la fabricación de gas mostaza y agentes neurotóxicos como el sarín y el vx. Además, había descubierto que el régimen de Sadam Husein había desarrollado un programa de armas biológicas que incluía agentes biológicos tan letales como el ántrax, la ricina o la toxina botulínica y agentes incapacitantes como micotoxinas o rotavirus. No obstante, tanto en lo que respecta a los agentes químicos como a los biológicos, en 2002 los iraquíes no habían logrado desarrollar dispensadores por aerosol susceptibles de ser transportados en medios aéreos capaces de causar bajas significativas en las tropas enemigas. En 1986, Estados Unidos bloqueó la condena de los ataques químicos de Irak contra Irán en el Consejo de Seguridad, siendo el único país que votó en contra de una resolución del Consejo de Seguridad que condenaba el uso de gas mostaza por parte de Irak contra las tropas iraníes.

18. Pastor, José Antonio: ¿Nuevo gendarme de la paz y seguridad internacionales? A propósito de la guerra de Irak de la primavera de 2003. Madrid: Edifer, Vol. 1, 2005, p. 467. 
de la fuerza como último recurso ${ }^{19}$. El 16 de marzo se reunieron el presidente George W Bush, el primer ministro Tony Blair y el presidente José María Aznar en la base de Lajes. Al día siguiente, este era el editorial del periódico The New York Times: «Estados Unidos, casi aislado, está a punto de librar una guerra en el nombre de la comunidad mundial que se opone a ella». Los dirigentes reunidos en las Azores quisieron arrogarse la representación de la comunidad internacional para darle un ultimátum no solo a Sadam Husein, sino al propio Consejo de Seguridad de la ONu, para al día siguiente, ante la perspectiva de lograr un mínimo apoyo internacional, poder pasar directamente a la guerra si Sadam Husein no dejaba el poder en 48 horas $^{20}$. El 20 de marzo de 2003, el día del inicio de la guerra, la Comisión de Inspección del Consejo del Consejo de Seguridad no había declarado el incumplimiento de Irak de la Resolución 1441. El 19 de marzo, el presidente de los inspectores, Hans Blix, había solicitado más tiempo para poder cumplir con su mandato ${ }^{21}$, en la sesión del Consejo de Seguridad. Estados Unidos y el Reino Unido, conscientes de la debilidad de su argumentario jurídico sobre la invasión de Irak, buscaban el apoyo de otros Estados, entre ellos España ${ }^{22}$. En Europa, excepción hecha del Reino Unido, de España y de un buen número de Estados del Este de Europa, la doctrina común partía de la imposibilidad de justificar el ataque a Irak, basándose en diversas resoluciones del Consejo de Seguridad, la 678 (1990), la 687 (1991) y la $1441(2002)^{23}$.

La Resolución 3314 (xxIx), de 14 de diciembre de 1974, aprobada por la Asamblea General de las Naciones Unidas, explicitaba de forma clara, en su artículo 2, que "el primer uso de la fuerza armada en contravención de la Carta" constituía "prueba prima facies de un acto de agresión». Estados Unidos, se mantenía en la consideración de que el uso de la fuerza estaba legitimado y autorizado por el Consejo de Seguridad, puesto que la autorización de 1990 no había finalizado, sino quedado en suspenso hasta su reactivación por la Resolución 1441, de 8 de noviembre de 2002, por la que se conminaba a Irak para que cumpliese las resoluciones de Naciones Unidas, bajo la amenaza de graves consecuencias para el régimen de Sadam Husein si continuaba poniendo en peligro la paz y la seguridad internacional ${ }^{24}$. La verificación del cumplimiento de las obligaciones impuestas a

19. JimÉNEZ, Carlos: «La política exterior española en torno a Irak y la relevancia del consenso en los ordenamientos internos e internacionales", en Cursos Derecho Internacional y Relaciones Internacionales de Vitoria-Gasteiz 2003, Vitoria: Servicio editorial de la Universidad del País Vasco, 2005. p. 284.

20. Ortega Klein, Andrés: "Naufragio en las Azores», Revista Elcano, 50, 2003, pp. 1-2.

21. El 14 de febrero de 2003, Blix presentó ante el Consejo de Seguridad un informe en el que aseguraba que Irak estaba en posesión de misiles de alcance superior a los $150 \mathrm{~km}$ autorizados. El 1 de marzo Irak comenzó la destrucción de los misiles Al Samoud 2, con el desmantelamiento de cuatro de ellos en la base militar de Al Tayi, al norte de Bagdad bajo supervisión de unMovic.

22. Remiro, Antonio: Derecho Internacional. Madrid: Tirant lo Blanch, 2007, p. 35

23. IOvame, Massimo y VitTor, Francesca de: "La doctrine europeénne et l'intervention en Iraq", Annuarie François de Droit International, núm. 49, 2003, p.10.

24. PÉrez, Guillermo Ángel: "Asia en el paso de un siglo a otro». En Díez, José Ramón et. al.: Historia del mundo actual: (desde 1945 hasta nuestros dias). Valladolid: Universidad de Valladolid, 2006, pp. 586-591. 
Irak en materia de desarme e inspección correspondía al mismo organismo que las había establecido, el Consejo de Seguridad, único órgano competente para aplicar nuevas sanciones y autorizar el uso de la fuerza, si consideraba que Sadam Husein no cumplía con lo establecido en la Resolución 687. Podría deducirse que la Resolución 1441 no autorizaba el uso de la fuerza contra Irak y que en el caso de que el Consejo de Seguridad lo considerase oportuno habría de emitir una nueva resolución que autorizase el uso de la fuerza de forma explícita. La realidad fue que esta resolución nunca existió y, por tanto, la invasión de Irak no se produjo al amparo de las Naciones Unidas ${ }^{25}$.

La posible posesión y empleo de armas de destrucción masiva por parte de Irak nunca fue probada ante el Consejo. La doctrina de "acción preventiva", en legítima defensa ante un supuesto ataque iraquí, tampoco legitimaba la invasión puesto que no se cumplía una premisa necesaria, el ataque previo al Estado interesado. La excusa de la «justa causa» tampoco podía validar un ataque bajo el pretexto de proteger los derechos humanos de una población sometida a una dictadura represora, ya que en el artículo 5.1 de la Resolución 3314 quedaba claro que ninguna consideración, fuera de carácter político, económico o militar, podía justificar una agresión. La propia Corte Internacional de Justicia había dictaminado que el empleo de la fuerza no podía constituir el método apropiado para verificar y asegurar el respeto de los derechos humanos. A pesar de lo expuesto, el Consejo de Seguridad, tras la invasión de Irak, no condenó la acción militar y llevó a cabo una política de hechos consumados, como demuestra el hecho de que asumiera la autoridad impuesta por las potencias ocupantes autorizando a una fuerza internacional, bajo el mando unificado de Estados Unidos, a adoptar las medidas oportunas para el mantenimiento en Irak de la seguridad y la estabilidad mediante la Resolución 1511, de 16 de octubre de $2003^{26}$. Podría parecer que Estados Unidos se sirvió de la onu para legitimar su acción en Irak y para dar apariencia de legalidad a prácticas dudosas en su política exterior.

Consumada la invasión, según el Proyecto de artículos de la Comisión de Derecho Internacional (CDI) ${ }^{27}$ sobre la responsabilidad del Estado por hechos internacionalmente ilícitos (2001), ningún miembro de la sociedad internacional debía reconocer la situación creada por la invasión, ni ayudar, en forma alguna, a su autor a consolidarla y cooperar para ponerla fin, si es que persistía en dicha actitud. La última de las obligaciones formaba parte del desarrollo progresivo del Derecho Internacional pero la primera ya constituía una parte fundamental del mismo

25. Calleo, David P.: Follies of power: America's unipolar fantasy. Cambridge: Cambridge University Press, 2009, pp. 55-57.

26. IGLESIAS, Alfonso Luciano: «La repercusión internacional de la invasión y reorganización política de Irak». En GuTiérrez, Ignacio (coord.), Irak. Invasión, ocupación y caos. Madrid: Los Libros de La Catarata, 2006, p. 75.

27. Órgano creado por la onu en 1947 para la codificación oficial del Derecho Internacional. 
general, como había puesto de manifiesto la Comisión de Derecho Internacional ${ }^{28}$. La ocupación de Irak se rigió en sus comienzos por el iv Convenio de la Haya de 1907 y Reglamento anexo, y por el Iv Convenio de Ginebra de $1949^{29}$. A estas normas del Derecho Internacional, se añadirían las resoluciones 1483 y 1511 (2003), y 1546 (2004) del Consejo de Seguridad.

La Resolución 1483 suponía la implicación de Naciones Unidas en la administración de una ocupación que no había autorizado ${ }^{30}$. No legalizaba la guerra, pero aceptaba una situación que violaba el Derecho Internacional. La Resolución 1546 (2004) aprobaba la formación de un Gobierno provisional soberano en Irak, presentado el 1 de junio de 2004, poniendo fin a la actividad de la Autoridad de la Coalición, que cesó en sus funciones el 30 de junio de ese año ${ }^{31}$. Esta Resolución revocaba el mandato de la Fuerza Multinacional, que había transmutado de fuerza ocupante a fuerza amparada por las resoluciones de Naciones Unidas por mediación de la Resolución 1511 (2003) ${ }^{32}$.

\section{ParticipaCión ESPAÑOla EN IRAK}

El 20 de marzo de 2003, fuerzas anglo-norteamericanas bombardeaban Bagdad sin la aprobación del Consejo de Seguridad de Naciones Unidas. Daba comienzo la operación Irak Freedom. Ese mismo día, el Gobierno español mostraba su total apoyo a la posición anglo-norteamericana y partía hacia Irak el primer contingente de tropas españolas. Comenzaba la operación Sierra Juliet ${ }^{33}$.

Las Fuerzas Armadas españolas participaron en dos misiones en Irak, la primera de ayuda humanitaria y la segunda de restablecimiento de la seguridad. La

28. GutiérRez, Cesáreo: La responsabilidad internacional (Consecuencias del hecho ilícito). Murcia: Diego Marín Librero, 2005, p. 60.

29. Convención IV relativa a las leyes y costumbres de la guerra terrestre y Reglamento anexo. Convenio Iv relativo a la protección de las personas civiles en tiempo de guerra.

30. SÁENZ, María Paz Andrés: «El Consejo de Seguridad en la guerra contra Irak: ¿ONG privilegiada, convalidador complaciente u órgano provisional?», Revista española de Derecho Internacional, núm. 55 , 2003, pp. 207-210. Por catorce votos a favor y la abstención de Siria, el Consejo de Seguridad reconocía la autoridad de Estados Unidos y el Reino Unido como potencias ocupantes, a los que denominaba «la Autoridad». El Consejo de Seguridad solicitaba al secretario general de Naciones Unidas que nombrase a un representante especial para Irak que tendría como misión coordinarse con la autoridad. De facto, las Naciones Unidas pasaban a colaborar con las potencias ocupantes (párrafo octavo).

31. Se estableció una estructura administrativa dual, formada por los órganos de la Coalición Internacional ocupante y elementos iraquíes designados por ella (la Asamblea y el Consejo de Gobierno Provisional) y una administración territorial internacional (la Misión de Asistencia de las Naciones Unidas para Irak).

32. Coma, Manuel: "La situación de Irak", Revista Elcano, núm. 3, 2003, pp. 4-7.

33. El ministro de Defensa denominó a la misión como S/J, utilizando el alfabeto fonético internacional. ¿Por qué Sierra Juliet?, la directiva que aprobaba el envío de la flota española a aguas del Golfo Pérsico se firmó el 19 de marzo, San José, S/J. El contingente formado por casi 900 soldados zarpó a las 10:30 horas del día 20 de marzo de 2003. 
primera misión fue autorizada mediante Acuerdo del Consejo de Ministros de 21 de marzo de 2003. La operación Sierra Juliet comenzó el 9 de abril de 2003, participaron el buque Galicia, la fragata Reina Sofía y el petrolero Marqués de la Ensenada. La inmediatez de la operación conllevó que no hubiese un plan definido, las tropas españolas se dirigían a Irak sin definir cuáles serían sus funciones, algo que se iría conociendo sobre la marcha. La travesía hacia el objetivo coincidió con la duración de los ataques a Irak. En Yibuti, se aprovisionó la flota del material ${ }^{34}$ que por la urgencia de su salida no había sido posible embarcar en España. El día 9 de abril de $2003^{35}$, el ministro de Defensa, Federico Trillo, informaba de que el buque anfibio, Galicia, había atracado en el puerto iraquí de Umm Qasr. Desde España se informó al contingente español en Umm Qasr de que, al día siguiente de su llegada, debía realizar la primera entrega de ayuda humanitaria. El reparto de ayuda fue conflictivo ${ }^{36}$ y las acciones posteriores se realizaron de forma más planificada y con mayor efectividad, en parte, debido al apoyo del imán chí, que ejercía cierto control sobre la población local. El reparto ${ }^{37}$ se centró en las familias más necesitadas, tras hacer un estudio previo de la población. Se repararon infraestructuras vitales.

Con el paso de los días, la misión española fue organizándose de forma más práctica. La unidad de guerra nuclear, química y biológica, (NBQ), resultó inútil para la misión a la que había sido encomendada, la búsqueda de armas de destrucción masiva, y fue utilizada en tareas más prácticas, como la seguridad y el apoyo en labores logísticas. El territorio de mayoría chii ${ }^{38}$ era un lugar donde reinaban la pobreza y la miseria, después de años bajo el régimen de Sadam Husein. Las calles no estaban asfaltadas, carecían de electricidad, de alcantarillado y de agua corriente, y los servicios sanitarios eran muy deficientes. Toda esta miseria contrastaba con la magnificencia del palacio de Sadam, en Basora. La primera misión centró su actividad en tareas de asistencia sanitaria, rehabilitación de escuelas, recogida de armamento y explosivos, y distribución de agua y alimentos entre la población iraquí. Se atendió a 5.238 pacientes, de los que 1.129 recibieron asistencia quirúrgica, se distribuyeron 14 toneladas de alimentos, 16 toneladas de agua embotellada, 20.000 litros de agua en aljibe, 5.000 litros de leche maternizada, 15.000 litros de

34. Material trasladado desde España por un avión de la Fuerza Aérea de España.

35. En España ya se estaba trabajando para desplazar a la zona nuevos efectivos. No sería hasta junio de 2003 cuando las primeras unidades de reconocimiento españolas se integrasen en las fuerzas de la operación Irak Freedom.

36. Las prisas por hacer la foto provocaron que se tuviera que suspender el reparto de ayuda humanitaria por los incidentes provocados por agitadores iraquíes.

37. Ya desde el primer reparto se ordenó resaltar las banderas españolas colocadas en las antenas de los vehículos militares, puesto que no se quería transmitir la imagen de una fuerza de ocupación, sino de un contingente que solo pretendía ayudar a la reconstrucción del país.

38. La ocupación del territorio se efectuó bajo dos doctrinas militares distintas, la británica, que intentó causar la menor destrucción posible en su operación de ocupación, y la estadounidense, contundente y demoledora. Silva, Lorenzo y Francisco, Luis Miguel: Y al final la guerra. La aventura de las tropas españolas en Irak. Madrid: Crítica, 2014, p. 56. 
aceite, 30.000 litros de leche en polvo, 1.440 litros de leche y otros productos. Los ingenieros del Ejército rehabilitaron la red de saneamiento de aguas de Umm Qasar, plantas potabilizadoras, una línea férrea y varias escuelas.

Los especialistas en explosivos levantaron un campo de minas, desactivaron 600 artefactos explosivos, inutilizaron 800 toneladas de explosivos y retiraron granadas de mortero y otros explosivos ${ }^{39}$. El 21 de junio, el contingente español partió de Umm Qasr. La operación Sierra Juliet había finalizado. El Consejo de Ministros español, en su reunión del 11 de julio de 2003, dispuso que España formase parte de la Misión Internacional que velaría por la seguridad y estabilidad del país hasta la celebración de elecciones democráticas y acordó el envío de tropas a Irak. Esta decisión se sustentaba, desde el punto de vista jurídico, en la Resolución 1483, que solicitaba a los Estados miembros de la onu su colaboración para crear las condiciones de seguridad necesarias en Irak, y en el artículo 97 de la Constitución española, que regula las competencias en materia de política exterior. La oposición acusó al Gobierno de José María Aznar de embarcar al país en una guerra sin cumplir lo dispuesto en el artículo 63 del texto constitucional.

No obstante, de las resoluciones 1483 y 1511 del Consejo de Seguridad se deducía que la guerra había terminado y que las acciones violentas perpetradas por elementos insurgentes en el país, eran actos terroristas y no acciones bélicas y por tanto, no sujetos al Derecho de Guerra. En consecuencia, si la situación en Irak, desde el punto de vista jurídico, no era de guerra, el envío de tropas, en respuesta a la llamada del Consejo de Seguridad, no podía interpretarse como que España enviase tropas a la guerra, sino como una misión que conforme a las citadas resoluciones, tenía como objetivo proporcionar seguridad y estabilidad a un país que debía encaminarse hacia un sistema democrático. Se autorizó el despliegue de la Brigada Plus Ultra $\mathrm{I}^{40}$, con un máximo de efectivos de 1.300. La zona asignada de despliegue fue el centro de Irak, An Nayaf y Al Nasiriyah. En esta segunda misión, España ejerció el mando de la Brigada Multinacional Plus Ultra I, formada por unidades de Nicaragua, El Salvador, Honduras y República Dominicana. La Brigada estaba integrada en la División Multinacional Centro-Sur ${ }^{41}$ Esta División, bajo mando polaco, estaba formada por 6.500 militares procedentes de Ucrania, Hungría, Mongolia, Filipinas, Rumanía, Eslovaquia, El Salvador, Honduras, Nicaragua, República Dominicana, España y Polonia. La Brigada Plus Ultra I controlaba dos

39. GutiérRez, Cesáreo y GonZÁlez, Andrés: El conflicto de Irak II, op. cit., p. 144.

40. La Brigada Plus Ultra I posibilitó el suministro de agua, electricidad y combustible a ciudades y pueblos, propició la puesta en marcha de hospitales, fábricas y escuelas, colaboró con las autoridades locales en la formación de ayuntamientos y juzgados, protección de instalaciones públicas, localización de enterramientos masivos y distribución de alimentos y medicinas, pagos de salarios a militares y funcionarios y colaboración en la formación de los cuerpos civiles de defensa iraquíes y en la seguridad de la población civil.

41. Las fuerzas de estabilización en Irak estaban constituidas por cuatro divisiones, dos norteamericanas en el norte y centro del país, una división británica en el sur y una multinacional en el centro-sur, en la que estaba integrada la Brigada española. 
provincias iraquíes, Al Qadisiya y An Nayaf, con una superficie de 8.153 y 28.824 $\mathrm{km}^{2}$ respectivamente, y cerca de 1.650 .000 habitantes. La nueva misión pasó a denominarse India Foxtrot que a diferencia de la operación Sierra Juliet, ya no tendría la ayuda humanitaria como objetivo principal, sino la seguridad, estabilidad y reconstrucción de Irak. En diciembre de 2003, llegó a Bagdad el primer avión español con ayuda humanitaria. La operación India Foxtrot se gestó mientras el contingente de la operación Sierra Juliet navegaba hacia el Golfo Pérsico. Desde un principio, el Plan de Operaciones del Jefe del Estado Mayor de la Defensa, (JEMAD), había concebido la misión española como una operación de mantenimiento de la $\mathrm{paz}^{42}$. No obstante, la operación en Irak iba a distar mucho de ser una operación de mantenimiento de la paz y fue evidente desde el mismo momento en el que las tropas españolas fueron atacadas el 20 de agosto de $2003^{43}$, cuando España estaba en proceso de relevo de las fuerzas estadounidenses en la zona asignada.

En Irak, hubo combates en toda regla ${ }^{44}$ en las condiciones de una guerra irregular y planteadas por una minoría insurgente, pero que hubo que afrontar por los medios y con la fuerza que requería el caso. Cualquier acción que se efectuaba en Irak estaba planificada y se ejecutaba en coordinación permanente con el responsable de la operación en Madrid. Tras el atentado en el que fallecieron siete agentes del Centro Nacional de Inteligencia, (CNI), Irak aparecía como un Estado peligroso donde había posibilidad de morir en combate ${ }^{45}$. Esa misión no iba a desarrollarse como en Kosovo o en Bosnia, el país árabe estaba sumido en el caos y no se respetaba al enemigo ni muerto por lo que la emboscada podía surgir en cualquier lugar. El día 28 de agosto de 2003, España se hacía cargo de la misión en la zona de Diwaniya y se producía la transferencia de autoridades con el batallón 3/5 de los Marines estadounidenses.

La toma de control de Nayaf se produjo el 23 de septiembre ${ }^{46}$. Cuando los españoles llegaron a Irak, la estructura de poder ya estaba definida. La organización

42. Las reglas de enfrentamiento eran claramente diferentes de las de otros países, como los Estados Unidos, y solo se contemplaba el uso de la fuerza en condiciones de legítima defensa. SiLva, Lorenzo y Francisco, Luis Miguel: Y al final la guerr, op. cit., p. 70.

43. El ataque a la Base España en Diwaniya evidenció que, para la insurgencia iraquí, los componentes de la Coalición eran objetivo militar independientemente de su nacionalidad.

44. En el transcurso de la Operación India Foxtrot, las tropas españolas en Irak sufrieron tres bajas militares. El 20 de agosto de 2003, fallecía el capitán de navío Manuel Martín-Oar en el ataque a la sede de la onu en Bagdad. El sargento primero, José Antonio Bernal Gómez, viceagregado del Centro Nacional de Inteligencia (CNI) en la Embajada española en Bagdad, fue asesinado en Bagdad el 9 de octubre de 2003, y el sargento Luis Puga Gandar, falleció a causa de un disparo accidental de un compañero el 26 de octubre de 2003.

45. Hasta el 4 de abril de 2004, en Nayaf, las tropas españolas destacadas en Irak tenían la impresión de que su misión era humanitaria y que el fin de esta era devolver a la normalidad a los habitantes de las ciudades de Diwaniya y Nayaf, dentro de la zona de responsabilidad del contingente español.

46. La zona bajo control español contaba con una población de más de dos millones de habitantes, la mayoría de confesión chií. Nayaf es considerada una ciudad santa para el chiismo. En ella se encuentra el segundo mayor cementerio islámico del mundo y es costumbre enterrar a los seres 
se había estructurado en dos ramas, la civil y la militar. En el ámbito militar, España estaba representada por el Cuartel General de Bagdad, el poder civil estaba representado por The Coalition Provisional Autbority (CPA), autoridad máxima civil en Irak en la que también había presencia de personal español. El primer contingente español de la operación India Foxtrot invirtió en su zona de responsabilidad una cifra cercana a los diez millones de dólares ${ }^{47}$. Se logró que las fábricas de Diwaniya volviesen a funcionar, se facilitaron los insumos necesarios para la actividad agrícola, se inició el curso escolar en octubre y se desarrolló una labor importante en el saneamiento del agua y el suministro de plantas potabilizadoras ${ }^{48}$. El 5 de septiembre de 2003, se ordenó al general Fulgencio Coll Bucher ${ }^{49}$ que preparase el nuevo contingente que habría de relevar a la Brigada Plus Ultra I, la Brigada Plus Ultra II, que tendría como núcleo de formación a la División Mecanizada. Antes de partir hacia el escenario de su misión ${ }^{50}$, se impartieron conferencias a las tropas en las que se formaban sobre aspectos de la cultura iraquí1 ${ }^{51}$, especialmente en lo que se refería a evitar actitudes que pudieran ser consideradas despreciativas por la población civil. El día 18 de diciembre de 2003, la Brigada Plus Ultra II estaba operativa en la zona asignada.

En lo que a la seguridad respecta, el contingente español debía hacer frente a la amenaza terrorista, que no siempre se correspondía con las acciones de la insurgencia iraquí, puesto que los mafiosos y bandoleros también hacían uso de técnicas terroristas. La manera en que la Brigada Plus Ultra II gestionaba su presencia en Irak basaba su fundamento en el concepto operativo que había guiado desde el comienzo la misión española y que no era otro que el que, en Irak, España desempeñaba una misión de mantenimiento de la paz en un escenario donde ya había finalizado la guerra. Los norteamericanos mantenían una visión muy diferente de su misión en Irak y continuaban aplicando métodos bélicos a su proceder, lo que

queridos en medio de grandes ceremonias, además de ser escenario de multitudinarias peregrinaciones. Para el creyente chí, no hay mayor honor que ser enterrado en la misma ciudad que Alí, primo del profeta y casado con su hija Fátima, además de primer imán del chiismo. El propio Alí había predicado que cualquier musulmán enterrado en Nayaf entraría en el paraíso.

47. Losada, Juan Carlos: Historia de las guerras de España. De la conquista de Granada a la guerra de Irak. Madrid: Pasado \& Presente, 2015, p. 851.

48. MarTínez, Eduardo: Recuerdos de Irak. Valladolid: AF Editores, 2004, p. 15.

49. Jefe de la Brigada de Infantería Mecanizada Extremadura XI.

50. El 15 de diciembre de 2003, la Brigada Plus Ultra II, al mando del general Coll, tomó el relevo de la Brigada Plus Ultra I, al mando del general Cardona Torres, en un acto presidido por el ministro Federico Trillo.

51. El Estado Mayor de la Defensa y el Estado Mayor Conjunto, la División de Inteligencia dependiente del Estado Mayor Conjunto, en su sección de Análisis y Alerta, redactó un informe llamado Básico Área Irak 2003-2004, en el que, entre otras cuestiones, se aconsejaba a las tropas españolas respetar las casas y las mezquitas, en caso de efectuar registros, no entrar sin permiso en las habitaciones de las mujeres ni utilizar perros, o evitar cacheos a mujeres por hombres, Además, se recomendaba vestir con decoro en un país con mayoría de población musulmana, no utilizar pantalones cortos ni camisetas de manga corta y, en el momento de entablar conversación, emplear palabras árabes e incluso entregar algún regalo. 


\section{JOSÉ ANTONIO LORENZO CUESTA \\ EL DIFÍCIL EQUILIBRIO ENTRE LA LEGALIDAD INTERNACIONAL Y LA POLÍTICA EXTERIOR: \\ LA PARTICIPACIÓN DE LAS FUERZAS ARMADAS ESPAÑOLAS [...] EN IRAK EN 2003}

ineludiblemente repercutía en el resto de los países participantes en la Coalición Internacional. A finales de febrero de 2004, el método español había conseguido que sus dos provincias de responsabilidad, Al Qadisiya y An Nayaf ${ }^{52}$, fueran zonas de relativa tranquilidad ${ }^{53}$ respecto del resto del país. El 20 de febrero de 2004, el general Ricardo Sánchez, máxima autoridad militar estadounidense en Irak, decidió intervenir en Nayaf, con el objetivo de cerrar los tribunales que aplicaban la Sharía, la ley islámica. El núcleo principal de la operación ${ }^{54}$ estaba constituido por unidades españolas y centroamericanas, además de personal de la CPA, policía iraquí, el Cuerpo de Defensa Civil iraquî́5 y la policía de mezquitas. El gran ayatolá de la mezquita advirtió a Muqtada Al-Sadar de que había instigado la ocupación de la mezquita ${ }^{56}$ por sus seguidores, sobre la posibilidad de solicitar la intervención de las fuerzas de la Coalición para desalojarlos si no desistían de su postura. Los grandes beneficios que proporcionaba la presencia en la Mezquita mediante la limosna eran razón suficiente para que los milicianos del Mahdí no abandonasen su posición.

En enero de 2004, desde el Cuartel General de la Coalición en Bagdad, se decidió poner fin a las actividades del clérigo chií. Se acordó que fuesen fuerzas iraquíes, con el apoyo de las tropas de la Coalición, las que pusieran fin a la presencia de los seguidores de Muqtada Al-Sadar en la Mezquita de Alí en Nayaf. Ante la negativa de las fuerzas iraquíes de intervenir en el desalojo de las huestes de Muqtada de la mezquita, el Cuartel General delegó esa misión en la Brigada Plus Ultra II. El general español, Coll Bucher, era consciente de que el mandato del Gobierno de España excluía cualquier tipo de operación ofensiva en Irak y tras consultar al Ministerio de Defensa, se transmitió a los mandos estadounidenses la negativa española a participar en la operación llevando el peso de la ofensiva, por lo que los planes estadounidenses fueron abandonados ${ }^{57}$. El día 3 de abril de 2004, fuerzas de operaciones especiales norteamericanas actuaron en Nayaf sin el conocimiento previo de

52. Al Qadisiya tenía una extensión de $8.153 \mathrm{~km} 2$ y 883.000 habitantes y su capital era Diwaniya. An Nayaf contaba con 931.600 habitantes repartidos en $28.824 \mathrm{~km} 2$ y con capital en Nayaf.

53. El 11 de febrero de 2004, una patrulla española en Diwaniya sufrió una emboscada por parte de los milicianos del Mahdí, que hostigaron por la noche a Base España con fuego de fusilería y mortero. Las órdenes de patrulla eran las de ejercer un control de su zona de responsabilidad en Diwaniya, obteniendo información e interesándose por las inquietudes de sus habitantes, mediante patrulla informativa a pie. Debían preocuparse especialmente por los puntos sensibles, fundamentalmente gasolineras y depuradoras de agua.

54. La operación fue bautizada con el nombre de Cordero Sagrado.

55. Creado por Orden n. ${ }^{\circ} 28$ de la CPA.

56. En enero de 2004, Muqtada Al-Sadar y sus acólitos del autodenominado ejército de al-Mahdi, habían intentado establecer, por la fuerza, un tribunal Sharía en la mezquita de Alí, en Nayaf, Tras un primer intento infructuoso el día 10 de enero, el 24 de ese mes consiguen hacerse con el control de una oficina dentro del edificio religioso.

57. Los militares españoles tenían la sensación de que su imagen ante los norteamericanos era la de un ejército débil y apocado. 
los mandos de la Brigada Multinacional Plus Ultra $\mathrm{II}^{58}$. Como resultado de esta operación, se detuvo al clérigo chií Mustafá Yaffa Al Yacubi, lugarteniente de Muqtada al-Sadar. Al Yacubi representaba la tendencia moderada del movimiento de al-Sadar y había desempeñado un papel importante en las negociaciones con la Brigada española y la policía iraquí, como representante de al Sadr. No sería hasta tres días más tarde cuando desde el Cuartel General de Bagdad, el comandante norteamericano responsable del área de operaciones aportó la información para la Brigada Plus Ultra II sobre la detención de Al Yacubi ${ }^{59}$. En Nayaf se culpaba a la Plus Ultra II de la detención del clérigo chií. También los medios de comunicación de Estados Unidos responsabilizaron a las tropas españolas de esta operación.

España era la responsable del arresto de Al Yacubi. La tarde del 3 de abril, cerca de medio millar de seguidores de al Sadr se concentraron a las puertas del destacamento español para exigir la liberación del detenido. De poco sirvieron las explicaciones del comandante Busquier, jefe de Asuntos Civiles de la Brigada, sobre la no responsabilidad de las tropas españolas en su detención y su presencia en la base. Los manifestantes impusieron un plazo de una hora para la liberación de Al Yacubi.

Las dos apacibles regiones hortofrutícolas que según el ministro Trillo los norteamericanos habían cedido a los españoles, se habían convertido, en un brevísimo espacio de tiempo, en zonas de conflicto. La labor encomiable ${ }^{60}$ de los españoles se había venido abajo en cuestión de horas por la intervención unilateral e inapropiada de Estados Unidos. Desde Bagdad, Kerbala, y Diwaniya fueron llegando autobuses repletos de radicales para exigir la liberación de Al Yacubi. A las 11 horas del día 4 de abril, la situación se tornó especialmente conflictiva: francotiradores del ejército de al-Mahdi tomaron posiciones en el Hospital Universitario de Nayaf, localizado en uno de los lados de la base Al Ándalus y que, por su altura, dominaba la zona ${ }^{61}$. Se produjo un enfrentamiento y los milicianos del ejército de al-Mahdi intentaron tomar la base española. Mediante su política de hechos consumados, los americanos habían arrojado a la Brigada española a

58. El día 2 de abril de 2004, la Brigada española había remitido, a España y al escalón superior en Irak, el Informe Diario de Situación, el International Military Staff Summary (INTSum), en el que se desaconsejaba cualquier acción punitiva, basándose en las diversas reuniones que se habían mantenido con líderes locales.

59. Antes de la llamada de los norteamericanos, la esposa de Al Yacubi ya se había presentado en la base Al Ándalus para solicitar su liberación, afirmando que los captores de su marido lucían uniformes y banderas españolas.

60. A finales de marzo de 2004, la División Multinacional Centro-Sur, en escrito dirigido a su Cuartel General, animaba a las brigadas polaca y ucraniana a seguir el modelo español de relación con las partes implicadas, pues era el único que estaba obteniendo resultados positivos. Boletín de Información Semanal, pp. 141-142. Este boletín era una especie de diario de la Brigada Plus Ultra II.

61. Los francotiradores abrieron fuego sobre el personal de la CPA que se encontraba en la azotea del edificio de la base Al Ándalus observando la manifestación. Un capitán estadounidense fue abatido y hubo varios heridos. 


\section{JOSÉ ANTONIO LORENZO CUESTA \\ EL DIFÍCIL EQUILIBRIO ENTRE LA LEGALIDAD INTERNACIONAL Y LA POLÍTICA EXTERIOR: \\ LA PARTICIPACIÓN DE LAS FUERZAS ARMADAS ESPAÑOLAS [...] EN IRAK EN 2003}

la guerra ${ }^{62}$. Esa tarde de abril, el teniente general Ricardo Sánchez, jefe supremo del Ejército norteamericano en Irak, se desplazó desde Bagdad para valorar la situación en Nayaf y se reunió con el general Coll Bucher. Al-Sadar, refugiado en la Mezquita de Kufa, animaba a sus seguidores a atacar a las fuerzas de la Coalición para liberar a Al Yacubi. La captura de Al Yacubi por los estadounidenses ocasionó la ruptura de relaciones con los representantes políticos, religiosos y sociales de las dos provincias bajo control español, puesto que los iraquíes consideraban que los españoles deberían haberles informado sobre la operación que condujo a la detención de Al Yacubi.

El Estado Mayor español estaba convencido de que la detención de Al Yacubi había dado al traste con el trabajo del contingente español en la zona ${ }^{63}$. El Mando americano preveía aumentar las operaciones de castigo en la zona bajo control español por lo que, en previsión del aumento de la actividad insurgente, se solicitó al Gobierno español el cambio en las reglas de enfrentamiento. El Ejecutivo español autorizó ese cambio permitiendo un uso mínimo de la fuerza solo en apoyo de las fuerzas de la Coalición que pudieran verse comprometidas en ataques ${ }^{64}$. Tras los atentados yihadistas, del 11 de marzo de 2004 en Madrid, el candidato socialista, José Luis Rodríguez Zapatero, anunció la retirada de las tropas españolas desplegadas en Irak si vencía en las elecciones que se celebrarían el 14 de ese mes ${ }^{65}$. El 11 de abril de 2004, los veteranos de la Plus Ultra II ya patrullaban juntamente con los recién llegados de las Plus Ultra ${ }_{\text {III }}^{66}$. El día 17 de abril, se produjo el relevo entre ambas brigadas al mando del general Muñoz, jefe de la Brigada de la Legión. El 18 de abril, José Luis Rodríguez Zapatero, en su toma de posesión como presidente del Gobierno español, anunció la decisión de retirar las tropas españolas de Irak a la mayor brevedad posible. La Brigada Plus Ultra III pasó a denominarse CONAPRE (Contingente de Apoyo al Repliegue).

62. Seis militares españoles fueron condecorados con la Cruz al Mérito Militar con distintivo rojo. Esta distinción solo se concede por acciones de fuerza armada en conflicto, en el que se han sostenido combates y causado bajas y que se ha acreditado el valor militar. El destacamento español de Nayaf sufrió, después del 4 de abril, veintiún ataques en quince días. En Diwaniya, se produjeron enfrentamientos entre milicianos seguidores de al-Sadar, atrincherados en la sede de Al Dawa, y las tropas españolas de Base España. Boletín de Información Semanal.

63. El Estado Mayor de la Defensa sabía muy bien en que terreno se habrían de mover las tropas españolas antes de su despliegue en Irak. El documento de Básico de Irak 2003-2004 señalaba que el sector Centro Sur, área asignada al contingente español, estaba habitado por una mayoría de población de credo chiita, una comunidad pobre pero hospitalaria, decía el informe, muy apegada a sus ancestrales tradiciones, muy devotos, seguidores de sus líderes religiosos y con un fortísimo sentimiento de comunidad. La inteligencia militar española había hecho sus deberes. El documento hacía una pormenorizada relación de la distribución de los grupos tribales en su zona de responsabilidad.

64. No se autorizaba el uso de la fuerza en operaciones ofensivas, en un momento en el que el Ejército de Estados Unidos había tomado la determinación de acabar con el poder del ejército de al-Mahdi, los milicianos de al-Sadar.

65. GutiérRez Espada, Cesáreo, González Martín, Andrés: El conflicto de Irak iI, op. cit., p. 148.

66. Sus efectivos pertenecían al Regimiento de Caballería Lusitania y estaban relevando al Regimiento de Caballería Farnesio. 
El Contingente ejecutó el repliegue en cuatro fases. En la primera fase, todo el contingente español se concentró en Diwaniya. En la segunda, se transfirieron las responsabilidades al $2 .^{\circ}$ Regimiento de Caballería de Estados Unidos ${ }^{67}$ y comenzó a replegarse el personal y material prescindible. En la tercera fase, se ejecutó el repliegue total y en la cuarta fase, se embarcó y trasladó todo el equipo a territorio nacional. Richard Myer, jefe del Estado Mayor Conjunto de las Fuerzas Armadas de Estados Unidos, acusó al pueblo español de haber cedido a las presiones del terrorismo yihadista, tras otorgar la victoria al Partido Socialista en las elecciones de marzo de $2004^{68}$. The New York Times dedicó su primera página a la retirada española de Irak en el mayor despliegue informativo sobre España desde 1939.

La decisión de retirar las tropas de Irak, por parte del Gobierno de Rodríguez Zapatero, no fue consensuada con el principal partido de la oposición y no obtuvo el respaldo de una resolución parlamentaria. Esta toma de postura unilateral afectó a la relación de España con Estados Unidos y pudo aparejar consecuencias significativas en cuatro ámbitos básicos de la política interior y exterior española.

La contrariedad de los Estados Unidos con el Gobierno español, tras la retirada de Irak, hizo temer que la cooperación en materia terrorista se viera afectada, en cuanto a la ayuda de los servicios de Inteligencia estadounidenses contra ETA; la hasta entonces convivencia pacífica de los intereses comerciales españoles y norteamericanos en Hispanoamérica; el papel de España como intermediario de los Estados Unidos en el Magreb y la complicada situación de la diplomacia española y los intereses de las grandes empresas españolas en el nuevo escenario geopolítico que habría de surgir en Oriente Medio, tras la caída del régimen de Sadam Husein $^{69}$. A los Estados Unidos no les molestó tanto la retirada como la manera de hacerlo, de forma un tanto precipitada, sin consenso con la Administración norteamericana y no gradualmente ${ }^{70}$. No obstante, los intereses mutuos y la postura española respecto de Afganistán, acercaron posturas entre el Gobierno socialista de España y la Administración Bush ${ }^{71}$.

67. El 20 de mayo de 2004 se arrió la bandera española en Diwaniya.

68. Chislett, William: "El antiamericanismo en España: el peso de la historia», Revista Elcano, 47, 2005, p.12.

69. Ruiz Miguel, Carlos: "La retirada española de Irak: significado y consecuencias", Revista Elcano, 81, 2004, pp. 3-5

70. Chilslett, William, «España y Estados Unidos: tan cerca y, sin embargo, tan lejos», Documentos de Trabajo (Real Instituto Elcano de Estudios Internacionales y Estratégicos), N. ${ }^{23}$, 2006, p. 6.

71. La decisión del Gobierno de Rodríguez Zapatero fue incrementar la presencia militar española en Afganistán y la contribución a la fuerza de la onu en el Líbano, con la quinta aportación de fuerzas más importante de las fuerzas integrantes de la misión a nivel internacional. Rodríguez Zapatero declaraba: «Estamos en Afganistán por las mismas razones por las que nos fuimos de Irak, por defender la paz, la onu y la legalidad internacional», una opinión que repitió el 7 de septiembre de 2006, día en que el Congreso aprobó el despliegue de tropas en el Líbano, cuando dijo: "Con los mismos principios y convicciones con que nos opusimos a la guerra de Irak y retiramos las tropas, las enviamos ahora al Líbano». Chilslett, William, "España y Estados Unidos: tan cerca y, sin embargo, tan lejos», Documentos de Trabajo (Real Instituto Elcano de Estudios Internacionales y Estratégicos), op. cit., p. 9. 


\section{JOSÉ ANTONIO LORENZO CUESTA \\ EL DIFÍCIL EQUILIBRIO ENTRE LA LEGALIDAD INTERNACIONAL Y LA POLÍTICA EXTERIOR: \\ LA PARTICIPACIÓN DE LAS FUERZAS ARMADAS ESPAÑOLAS [...] EN IRAK EN 2003}

En las elecciones de marzo de 2004, dos posturas divergentes se habían enfrentado sobre la concepción de lo que debía ser la actuación del Gobierno en materia de relaciones exteriores, el Partido Popular, inspirado por José María Aznar, consideraba que el Gobierno debía dirigir a la opinión pública en política exterior; Rodríguez Zapatero pensaba que la opinión pública marcaba el ritmo en esa materia. La retirada de Irak y el aumento de la presencia militar española en Afganistán ponían de manifiesto que el Gobierno socialista español consideraba la misión en Afganistán una acción respaldada por la Comunidad Internacional contra un país que era considerado como base de entrenamiento de terroristas. Irak, sin embargo, era visto más como un obstáculo en la lucha contra el terrorismo islámico.

\section{CONClusiones}

La visión que José María Aznar tenía del desempeño de España en política exterior fue un factor decisivo en su incondicional apoyo de la postura de los Estados Unidos en Irak, su objetivo era hacer del país la nación que fue antaño, un país grande $^{72}$ en el concierto internacional. En España, el Parlamento y la sociedad, en su mayoría, mostraron su reticencia a establecer una política de estrecha colaboración con los Estados Unidos en materia de relaciones exteriores. A pesar de los esfuerzos del Gobierno Aznar, España carecía del potencial económico, político, militar y diplomático necesario para persuadir a sus socios europeos de las «bondades» de una intervención militar en Irak liderada por Estados Unidos ${ }^{73}$. España perdió el tradicional papel de mediador con las naciones árabes y parte de su influencia en el ámbito de la Unión Europea, en un escenario en el que resultaba fundamental que España dispusiera de la capacidad necesaria de negociación para no perder influencia, en una etapa en la que la ampliación de la Unión Europea podía alejar a España del núcleo principal de la toma de decisiones ${ }^{74}$.

Dado el carácter particular de la política exterior del Gobierno Aznar, se evidenciaba que cuando cesase en su cargo, su sucesor habría de introducir cambios sensibles en esta materia. Cuando el cambio se produjo a favor de otro partido

72. «España tiene todas las potencialidades para ser una de las democracias más importantes de Europa y del mundo. España tiene hoy una consideración y respeto internacional y puede aumentar su presencia en el mundo. España debe llegar al tren de la historia a tiempo. La nueva España puede llegar a tener una posición más fuerte en el escenario internacional. Tenemos que hacer muchas cosas en el exterior. Tenemos que aprovechar muchas oportunidades", José María Aznar en El País, 8-4-2003.

73. Tusell, Javier: El Aznarato. El Gobierno del Partido Popular 1996-2004. Madrid: Aguilar, 2004, p. 337.

74. José María Aznar deseaba para España una relación con Estados Unidos similar a la que tenía el Reino Unido. El presidente Aznar había llegado a la conclusión de que una relación privilegiada con Washington reforzaría el papel de España en Europa, que había visto como el eje franco alemán había eclipsado al resto de los países de la Unión Europea, incluida España. Que la Administración norteamericana estaba dispuesta a otorgar ese papel a España lo demuestra el hecho de que el presidente Bush eligiese España como el primer país en la escala en su primera visita a Europa, en junio de 2001. 
político y de otro presidente de Gobierno, la línea de actuación en materia de relaciones exteriores ${ }^{75}$ del nuevo Ejecutivo dio un giro de $180^{\circ}$ y la materialización de este cambio fue la retirada de las tropas españolas de $\operatorname{Irak}^{76}$. Múltiples teorías se han aportado sobre el verdadero trasfondo de la ocupación de Irak por Estados Unidos: desde un supuesto interés en controlar los recursos petrolíferos del país, pasando por la consideración de un nuevo ejemplo de neocolonialismo, hasta el resultado de la lucha de intereses de las grandes multinacionales ${ }^{77}$. Seguramente, todos estos factores influyeron a la hora de adoptar la decisión de derrocar a Sadam Husein, aunque tal vez, fueran los intereses geoestratégicos de la nueva potencia hegemónica los verdaderos impulsores de la ocupación de $\operatorname{Irak}^{78}$.

La guerra de 2003 no fue solo generada por factores tan importantes como el petróleo o por la inestabilidad en Próximo Oriente. Tampoco se llevó a cabo con la prioridad de liberar al pueblo iraquí o liberar al mundo de la carga de las armas de destrucción masiva. Más bien, al igual que la operación efectuada en Afganistán, la guerra de Irak supuso una clara demostración de que Estados Unidos, tras el 11S, disponía de la capacidad y de la voluntad de derrotar a los Estados «supuestamente poderosos» y de frenar los peligros a los intereses vitales del pueblo estadounidense ${ }^{79}$.

La Carta de Naciones Unidas de 1945 prohíbe a los Estados el uso de la fuerza en las relaciones internacionales salvo en caso de legítima defensa. Sin embargo, este argumento ${ }^{80}$ no puede ser invocado en el caso de Irak, al referirse al uso de la fuerza armada por un Estado víctima de un ataque de envergadura llevado a cabo por las fuerzas armadas de otro Estado que penetren en su territorio, lo bombardeen o ataquen a sus fuerzas terrestres, navales o aéreas, o a su flota mercante o área civil, o por grupos armados «enviados» por un Estado para cometer, en otro, actos de gravedad equiparables a los mencionados o respecto de cuyos actos un

75. En una entrevista concedida al diario norteamericano, The Wall Street Journal, José María Aznar advertía de que la retirada de las tropas españolas de Irak supondría para España una dificultad en sus relaciones exteriores. The Wall Street Journal, 26 de abril de 2004. Durante el Gobierno de Rodríguez Zapatero, se incrementó la presencia militar española en Afganistán y la contribución española a la fuerza de la onu en el Líbano. Rodríguez Zapatero justificaba la presencia española en Afganistán por las mismas razones que había retirado las tropas de Irak, defender la paz, la onu y la legalidad internacional.

76. ARENAL, Celestino del: «La retirada de las tropas de Irak y la necesidad de una nueva política exterior", Revista Elcano, 82, (2004), p. 3. La situación en Irak se había agravado una vez finalizadas las operaciones bélicas. En marzo de 2004, las Naciones Unidas tendrían que renunciar al control político y militar de Irak a favor de Estados Unidos. Este hecho venía a respaldar, ante la opinión pública española, ya mayoritariamente contraria a la participación de España en Irak, la decisión de retirar las tropas a la mayor brevedad posible del avispero en el que se había convertido Irak. Esta decisión conllevó un enfriamiento de las excelentes relaciones que habían mantenido el Gobierno Aznar y la Administración Bush.

77. RAI, Milan: Plan de guerra contra Iraq. Diez razones para no iniciar una nueva guerra contra Iraq. Madrid: Foca, 2003, pp. 54-56

78. Prieto, Mónica G. y Espinosa, Javier: La semilla del odio. De la invasión de Irak al surgimiento del Isis. Barcelona: Debate, 2017, pp. 18-21.

79. Williamson, Murray y Scales, Jr., Robert, H.: The Irak War. A military bistory. Nueva York: Harvard University Press, 2003, pp. 251-252.

80. Artículos 24 y 51. 


\section{JOSÉ ANTONIO LORENZO CUESTA \\ EL DIFÍCIL EQUILIBRIO ENTRE LA LEGALIDAD INTERNACIONAL Y LA POLÍTICA EXTERIOR: \\ LA PARTICIPACIÓN DE LAS FUERZAS ARMADAS ESPAÑOLAS [...] EN IRAK EN 2003}

Estado, que no ha enviado a esos grupos, "participe sustancialmente» ${ }^{81}$. Ninguna de estas condiciones se cumplió en el caso iraquí. Irak no atacó con su ejército a Estados Unidos, ni envió grupos armados para cometer actos de gravedad equiparables, ni participó sustancialmente en actos armados de este tipo cometidos por grupos armados existentes en Estados Unidos ${ }^{82}$. En este contexto, la invasión de Irak por parte de Estados Unidos y el Reino Unido supuso la vulneración del ius $\operatorname{cogens}^{83}$. Tras la finalización de la guerra, extensos territorios de Irak se convirtieron en feudos de grupos de la insurgencia en los que la ley la imponían los señores de la guerra y no el Gobierno provisional con las fuerzas de la Coalición.

Los atentados contra las tropas de la Coalición presentes en suelo iraquí respondían respondieron más a las condiciones de la ocupación y la degradación de la vida del pueblo iraquí ${ }^{84}$ que a un plan organizado por parte del terrorismo internacional o elementos del régimen iraquí contra la nueva situación derivada de la ocupación. Si, desde el punto de vista jurídico, el envío de tropas españolas a Irak podía considerarse conforme a Derecho, desde el punto de vista de la política, la realidad fue muy distinta. El principal partido de la oposición, el Partido Socialista, con su líder José Luis Rodríguez Zapatero, había mostrado su desacuerdo con el envío de tropas a Irak y defendía que esta postura involucraba a España en una guerra sin el apoyo de la opinión pública.

Para el Partido Socialista, la fuerza multinacional presente en Irak debía permanecer bajo el control político y militar de la ONU como condición para considerar aceptable el envío de tropas a ese país. Sin embargo, en las resoluciones de la Naciones Unidas se encargaba a Estados Unidos y al Reino Unido el control territorial de Irak hasta su estabilización. El 18 de abril de 2004, una vez investido como nuevo presidente por las Cortes Generales, José Luis Rodríguez Zapatero anunció en televisión la inmediata retirada de las tropas españolas de Irak, sin esperar a

81. Art. 51 de la Carta de Naciones Unidas y art. 3, letras a, b, c y g de la Definición de Agresión aneja a la Resolución 3314 (xxix) adoptada el 14 de diciembre de 1974 por la Asamblea General de Naciones Unidas.

82. MCGOLDRICK, Dominic: From 9-11 to the Iraq War. International law in an age of complexity. Nueva York: Hart Publishing, 2004, p. 83.

83. Picone, Paolo: "La Guerra contro l'Iraq e le degenerazioni dell'unilateralismos», Rivista di Diritto Internazionale, 86, 2003, p. 370.

84. Una encuesta de la British Broadcasting Corporation (BBC), de agosto de 2005, mostraba que un $82 \%$ de los iraquíes eran contrarios a la presencia de tropas de la Coalición en su país. Un $72 \%$ declaraba no tener ninguna confianza en esas fuerzas internacionales y solo un $1 \%$ se mostraba satisfecho con la actuación de las tropas de la Coalición. En marzo de 2004, Paul Bremer firmó personalmente la orden de cierre del semanario Al-Hawza al-Natiqa, publicación cercana a la corriente del clérigo Muqtada al-Sadar. El semanario criticaba el estado de abandono de las infraestructuras del sur del país y la dejadez para resolver los problemas de los ciudadanos, por parte de las autoridades de la Coalición y las nuevas autoridades iraquíes. Después de la guerra de 20031, la descapitalización de la economía iraquí había afectado sensiblemente a las capas más vulnerables de la sociedad. En la década de los ochenta del siglo xx, el Producto Interior Bruto (PIB) de Irak se estimaba en 3.500 \$, en el año 2001, después de años de embargo, esa cifra se había reducido hasta los 866 \$. Gutiérrez Espada y Cesáreo, GonZÁlez MARTín, Andrés: El conflicto de Irak II, op. cit., p. 141. 
JOSÉ ANTONIO LORENZO CUESTA

EL DIFÍCIL EQUILIBRIO ENTRE LA LEGALIDAD INTERNACIONAL Y LA POLÍTICA EXTERIOR:

LA PARTICIPACIÓN DE LAS FUERZAS ARMADAS ESPAÑOLAS [...] EN IRAK EN 2003

la fecha del 30 de junio anunciada previamente. La decisión fue tomada de forma unilateral por el nuevo presidente ${ }^{85}$, sin consultar al Partido Popular ni debatirla en el Parlamento español. Quedaba en evidencia que la política exterior sin consenso estaba condenada a variar al ritmo del cambio de los respectivos gobiernos surgidos de los procesos electorales. De haberse consensuado por parte del Gobierno del Partido Popular la forma y modo de participación española en Irak en 2003 el resultado no hubiese sido la retirada abrupta de las tropas españolas de Irak con la llegada al poder del Gobierno de José Luis Rodríguez Zapatero.

Esta decisión provocó consecuencias previsibles en el ámbito de las relaciones diplomáticas de España, especialmente con los Estados Unidos, que había manifestado al Gobierno español su deseo de que el contingente español permaneciese en Irak $^{86}$. José María Aznar había adoptado una nueva línea en materia de relaciones exteriores sin el consenso del resto de las fuerzas políticas del arco parlamentario, algo que había sido habitual desde la Transición. En resumen, un Gobierno legítimo y democrático, el de José María Aznar, adoptó una decisión en materia de política exterior involucrando al Estado español, a sabiendas de que la ciudadanía se oponía a la participación en la guerra de Irak sin el consentimiento de la onu. España, con su precipitada retirada de Irak, pagó un alto precio en prestigio internacional, ganado durante muchos años de política exterior consensuada en el Parlamento. Si a la hora de dar luz verde a la participación española en Irak, el Gobierno del Partido Popular actuó guiado por la ideología, no lo fue menos en el caso del Gobierno de José Luis Rodríguez Zapatero. El Ejecutivo del Psoe había ganado las elecciones del año 2004 con la promesa de retirar el contingente español de Irak si la onU no «legitimaba su presencia en ese país", mediante el control militar de todas las tropas allí presentes, una entelequia desde el punto de vista militar y de política exterior. Las Naciones Unidas habría legalizado la ocupación militar de Estados Unidos y Reino Unido en Irak, y las tropas que allí operaban estarían bajo el paraguas legal de la onU, mediante la Resolución 1483. La ONU no cambió de postura respecto a Irak y el Gobierno español ordenó la retirada inmediata de las tropas españolas con el consiguiente perjuicio para el prestigio de España en materia de misiones militares: España dejó de ser un socio fiable para muchos países aliados. Los militares españoles se desplegaron cuando se les ordenó, cumplieron con un alto nivel de profesionalidad su misión y regresaron en el momento en que el Gobierno lo consideró oportuno. Probablemente, los únicos beneficiados de esta aventura exterior de España fueron los miles de ciudadanos iraquíes a los que las tropas españolas llevaron ayuda en una situación de especial gravedad en su país.

85. Para Rodríguez Zapatero, el Partido Popular y José María Aznar habían acabado con el modelo de política exterior español, consensuado desde 1976, y era urgente recuperar esta línea de actuación de nuevo para que España pudiera ejercer de árbitro en las complicadas relaciones exteriores de Próximo Oriente. ARENAL, Celestino del: «La retirada de las tropas de Irak y la necesidad de una nueva política exterior», op. cit,. p. 1.

86. RuIz, Carlos: «La retirada española de Irak: significado y consecuencias, op. cit., p. 4. 